Article

\title{
Synthesis and Antitumor Evaluation of Novel 5-Hydrosulfonyl-1H-benzo[d]imidazol- 2(3H)-one Derivatives
}

\author{
Guang Ouyang ${ }^{1,+}$, Rongsheng Tong ${ }^{1,+}$, Jinqi Li ${ }^{1}$, Lan Bai ${ }^{1}$, Liang Ouyang ${ }^{3}$, Xingmei Duan ${ }^{1}$, \\ Fengqiong $\mathrm{Li}^{1}{ }^{1}$, Pin $\mathrm{He}^{1}$, Jianyou Shi ${ }^{1, *}$ and Yuxin $\mathrm{He}^{2, *}$ \\ 1 Individualized Medication Key Laboratory of Sichuan Province, \\ Sichuan Academy of Medical Science \& Sichuan Provincial People's Hospital, \\ Chinese Academy of Sciences Sichuan Translational Medicine Research Hospital, School of Medicine, \\ Center for Information in Medicine, University of Electronic Science and Technology of China, \\ Chengdu 610072, Sichuan, China; oyg613@163.com (G.O.); tongrs@126.com (R.T.); \\ lijinqi2002@126.com (J.L.); blci@163.com (L.B.); duanxingmei2003@163.com (X.D.); \\ lifengqiong2014@163.com (F.L.); hep403@163.com (P.H.) \\ 2 Bioengineering College, Xihua University, Chengdu 610039, Sichuan, China \\ 3 State Key Laboratory of Biotherapy, West China Hospital, Sichuan University, Chengdu 610041, Sichuan, \\ China; klivis@163.com \\ * Correspondence: shijianyoude@126.com (J.S.); heyuxin66@126.com (Y.H.); \\ Tel.: +86-177-0813-0657 (J.S.); +86-139-8185-0645 (Y.H.) \\ + These authors contributed equally to this work.
}

Academic Editor: Jean Jacques Vanden Eynde

Received: 26 January 2016; Accepted: 31 March 2016; Published: 20 April 2016

\begin{abstract}
A series of novel 5-hydrosulfonyl-1H-benzo[d]imidazol-2(3H)-one derivatives bearing natural product substructures has been successfully synthesized and their antitumor activity studied. These newly synthesized derivatives were characterized by ${ }^{1} \mathrm{H}-\mathrm{NMR},{ }^{13} \mathrm{C}-\mathrm{NMR}$ and high resolution mass spectral data, then screened as antitumor agents against the A549, HCC1937, and MDA-MB-468 human tumor cell lines using MTT cell proliferation assays. The results show that some of these compounds can effectively inhibit the growth of these cancerous cells, with compound $\mathbf{5 b}$ being the best one $\left(\mathrm{IC}_{50}=2.6 \mu \mathrm{M}\right)$. Flow cytometry data revealed that compound $5 \mathbf{b}$ induced apoptosis of HCC1937 cells with increased solution concentration. The structure and activity relationships (SAR) of these compounds is summarized.
\end{abstract}

Keywords: antitumor activity; 5-hydrosulfonyl-1H-benzo[d]imidazol-2(3H)-one; synthesis

\section{Introduction}

Cancer poses a threat to worldwide health due to its high mortality. Environmental degradation has been associated with increased cancer incidence [1]. It is estimated that the global incidence of cancer in both men and women will increase from an estimated 12.7 million cases in 2008 to 20.3 million by 2030 , a net increase of about $60 \%$ [2]. Therefore, it is very urgent to develop new, high effective and safe antitumor drugs.

Dibromophakellstatin (Figure 1), a natural product isolated from Phakellia mauritiana, has good antitumor activity [3]. Dibromophakellstatin, a new cancer cell growth inhibitor, has a dihydrobenzoimidazol-2-one scaffold [4]. Other dihydro-benzoimidazol-2-one analogues have been found to be effective compounds when used as hypoglycemia agents [5], muscarinic acetylcholine esterase inhibitors [6], antimicrobial agents [7] and pigments [8]. In recent years, dihydro-benzoimidazol2-one, was used as a novel scaffold in the tumor therapeutic area, and attentions on it has been increasing. Dihydrobenzoimidazol-2-one derivatives show increased anti-tumor activity by inhibiting 
different receptors [9-14], now there has been great progress in the study of dihydro-benzoimidazol2-one derivatives. Thus, halopemide [15] (Figure 1) is the first potent, direct-acting, drug-like, small-molecule phospholipase D1/2 (PLD1/2) inhibitor; UV01555069 and UV0359595 are potent selective phospholipase D1 (PLD1) inhibitors [16,17]; 5-(5-((2-aminoethyl)amino)pyrazolo[1,5-a] pyrimidin-3-yl)-1H-benzo[d]imidazol-2(3H)-one is a Pim-1/2 kinase inhibitor [18]. Other effects, such as reversible Cdc25 inhibition [19]; dual CCR3/H1 antagonism [20]; and non-thiolfarnesyltransferase inhibition [21] are also reported. In the above studies, structural modifications were mainly performed at the 1-, 3- and 5-positions of the dihydrobenzoimidazol-2-one ring. The sulfonamide group is an important pharmacophore that often appears in antitumor drugs. E7010 [22], for example, is a kind of tubulin inhibitor containing a sulfonamide group and it was shown that the sulfonamide group played an important role in its activity. To the best of our knowledge, however, the replacement of a sulfamide group at the 5-position of the dihydrobenzoimidazol-2-one scaffold is not reported.<smiles>O=C1CC2n3c(cc(Br)c3Br)C(=O)N3CCCC23N1</smiles>

Dibromophakellstatin KM2OL2 ED $50=0.11 \mathrm{ug}$ SK-MEL-5 ED $50=0.11 \mathrm{ug}$<smiles>COC(=O)OCCOc1ccccc1</smiles>
UV0155069 PLD1 IC $50=11 \mathrm{nM}$ PLD2 IC ${ }_{50}=1800 \mathrm{nM}$<smiles>NCCNc1ccn2ncc(-c3ccc4[nH]c(=O)[nH]c4c3)c2n1</smiles>

5-(5-((2-aminoethyl)amino)pyrazolo[1,5-a] pyrimidin-3-yl)-1H-benzo[d]imidazol-2(3H)-one Pim1 $\mathrm{IC}_{50}=7.2 \mathrm{nM}$ Pim $2 \mathrm{IC}_{50}=19 \mathrm{nM}$

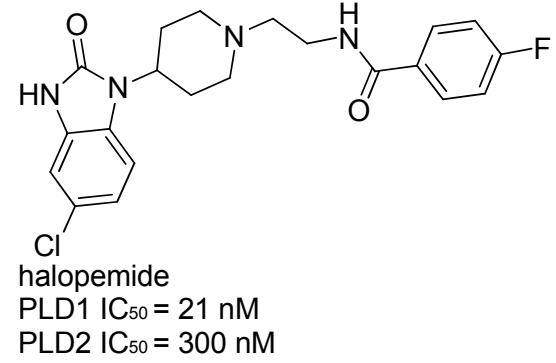<smiles>CC(CN1CCC(n2c(=O)[nH]c3cc(Br)ccc32)CC1)NC(=O)[C@@H]1C[C@H]1c1ccccc1</smiles>

UV0359595

PLD1 IC $50=3.7 \mathrm{nM}$

PLD2 IC 50 $_{0}=6400 \mathrm{nM}$<smiles>COc1ccc(S(=O)(=O)Nc2cccnc2Cc2ccc(O)cc2)cc1</smiles>

E7010

Figure 1. Structures and activities of dibromophakellstatin $1 \mathrm{~A}$, halopemide $2 \mathrm{~A}$, isoform-selective PLD (PLD1 selective) inhibitors 3A and 4A, Pim 1/2 inhibitor 5-(5-((2-aminoethyl)amino)pyrazolo [1,5-a]pyrimidin-3-yl)-1H-benzo[d]imidazol-2(3H)-one, tubulin inhibitor E7010.

Because dihydrobenzoimidazol-2-one derivatives have potential antitumor activity, we focused our attention on introducing proper functional groups into the dihydrobenzoimidazol-2-one ring. Previous studies on the dihydrobenzoimidazol-2-one scaffold showed that such compounds have potent antitumor activity, so in this work a series of 5-hydrosulfonyl-1H-benzo[d]imidazol-2(3H)-one derivatives has been designed and synthesized by introducing the sulfamide groups at the 5-position. The new compounds were tested for their anti-cancer activity against some different cell lines and it was found that compound $\mathbf{5 b}$ (Table 1) has the best activity against the HCC1937 cell line. 
Table 1. In vitro cytotoxic activity of target compounds: $\mathbf{5 a - o .}$<smiles>[R1]Cn1c(=O)[nH]c2cc(S([R])(=O)=O)ccc21</smiles>

$\mathrm{IC}_{50}(\mu \mathrm{M})$

\begin{tabular}{|c|c|c|c|c|c|}
\hline No. & Compound & MDA-MB-48 & A549 & HCC1937 & Hek293 \\
\hline 1 & $5 a$ & $9 \pm 0.67$ & $5.2 \pm 0.35$ & $7.8 \pm 0.49$ & $>10,000$ \\
\hline 2 & $5 b$ & $6.3 \pm 0.45$ & $3.1 \pm 0.17$ & $2.6 \pm 0.21$ & 292.6 \\
\hline 3 & $5 c$ & $>200$ & $88 \pm 6.1$ & $105 \pm 8.9$ & 248.2 \\
\hline 4 & $5 d$ & $73 \pm 6.9$ & $77 \pm 6.8$ & $85 \pm 7.1$ & 756.4 \\
\hline 5 & $5 e$ & $>200$ & $>200$ & $127 \pm 9.9$ & $>10,000$ \\
\hline 6 & $5 f$ & $82 \pm 6.1$ & $>200$ & $>200$ & $>10,000$ \\
\hline 7 & $5 \mathrm{~g}$ & $>200$ & $>200$ & $152 \pm 9.8$ & 2000.9 \\
\hline 8 & $5 \mathrm{~h}$ & $>200$ & $>200$ & $>200$ & 369.7 \\
\hline 9 & $5 i$ & $>200$ & $>200$ & $>200$ & 708.9 \\
\hline 10 & $5 j$ & $>200$ & $>200$ & $>200$ & 665.9 \\
\hline 11 & $5 \mathrm{k}$ & $135 \pm 9.7$ & $116 \pm 7.6$ & $>200$ & 383.6 \\
\hline 12 & 51 & $22 \pm 1.6$ & $15 \pm 1.1$ & $18 \pm 1.2$ & 6312.3 \\
\hline 13 & $5 \mathrm{~m}$ & $>200$ & $>200$ & $>200$ & 447.6 \\
\hline 14 & $5 n$ & $12 \pm 1.02$ & $16 \pm 0.9$ & $22 \pm 1.3$ & $>10,000$ \\
\hline 15 & 50 & $15 \pm 0.93$ & $9.7 \pm 0.88$ & $11 \pm 0.71$ & $>10,000$ \\
\hline \multicolumn{2}{|c|}{ Doxorubicin } & $2.7 \pm 0.19$ & $2.5 \pm 0.13$ & $4.8 \pm 0.22$ & 12.2 \\
\hline
\end{tabular}

\section{Results and Discussion}

\subsection{Chemistry}

The novel 5-hydrosulfonyl-1H-benzo[ $d]$ imidazol-2(3H)-one derivatives described herein were synthesized as shown in Scheme 1. Substitution reactions of 2-nitroaniline (1) with benzyl bromide or (2-bromoethyl)benzene and $\mathrm{NaOH}$ in acetone gave compounds 2, then chlorosulfonation with chlorosulfonic acid and another substitution reaction with ammonia derivatives in tetrahydrofuran (THF) gave compounds 3 , which were reduced by $\mathrm{Fe} / \mathrm{HCl}$ to afford compounds 4 , which were then cyclized with triphosgene (BTC) in anhydrous THF to give compounds $\mathbf{5 a - o}$. All the newly synthesized compounds were characterized on the basis of their ${ }^{1} \mathrm{H}-\mathrm{NMR},{ }^{13} \mathrm{C}-\mathrm{NMR}$ and HRMS data.

\subsection{Anti-Cancer Activity}

Compounds 5a-o were evaluated for their cytotoxic activity in vitro against some human cancer cell lines, including A549, HCC1937 and MDA-MB-468 by applying a MTT colorimetric assay. Doxorubicin was used as positive control. The calculated $\mathrm{IC}_{50}$ values were reported differently according to the different cancer cells. The results are summarized in Table 1. The data in Table 1 shows that some of the synthesized compounds exhibited distinct cytotoxic activity against the A549, HCC1937 and MDA-MB-468 human cancer cell lines in vitro. 
<smiles>[R]S(=O)(=O)c1ccc(NC(=O)c2ccccc2)c([N+](=O)[O-])c1</smiles><smiles>[R]S(=O)(=O)c1ccc(NC(C)C)c(NC(C)C)c1</smiles>

$5 a \sim j, n=1$

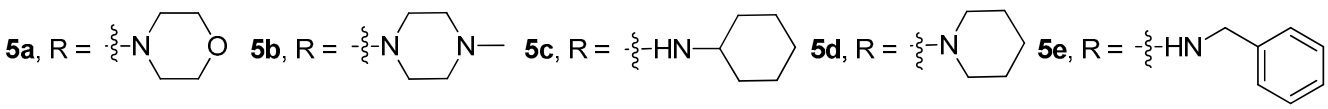

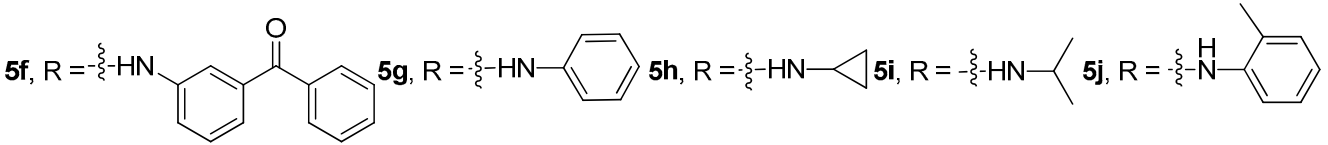

$5 k \sim 0, n=2$

$5 k, R=\xi-H N \longrightarrow$

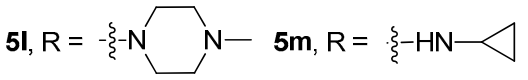

$5 n, R=-\xi-N$

5o, $R=-\frac{\xi}{2}+\mathrm{O}$

Scheme 1. Syntheses of compounds 5a-o. Reactions and conditions: (a) $\mathrm{NaOH}$, benzyl bromide or (2-bromoethyl)benzene, acetone, $65^{\circ} \mathrm{C}, 1.5 \mathrm{~h}$; (b) chlorosulfonic acid, $0{ }^{\circ} \mathrm{C}, 4 \mathrm{~h}$; (c) ammonia derivatives, $\mathrm{THF}, \mathrm{rt}, 0.5 \mathrm{~h}$; (d) Fe/ HCl, silica gel, $80 \%$ ethyl alcohol, $80{ }^{\circ} \mathrm{C}, 1 \mathrm{~h}$; (e) triphosgene, anhydrous THF, $0{ }^{\circ} \mathrm{C}, 0.5 \mathrm{~h}$.

\subsection{Flow Cytometry}

To exhibit a far more explicit function of compound $\mathbf{5 b}$ on HCC1937 Cells, we did a cell flow experiment with HCC1937. When the concentration of $5 \mathbf{b}$ was $7.5 \mu \mathrm{M}$, the proportion of apoptotic cells was $11.4 \%$. When the concentration was $10 \mu \mathrm{M}$, the proportion of apoptotic cells was $13.4 \%$. When the concentration was $15 \mu \mathrm{M}$, the proportion of apoptotic cells was $12.9 \%$. When the concentration was $20 \mu \mathrm{M}$, the proportion of apoptotic cells was $29 \%$. The proportion of apoptotic cells in control group was $6.7 \%$. As the concentration increased, the number of apoptotic cells increased gradually and the number of apoptotic cells and concentration were directly proportional, although there was no significant difference between $10 \mu \mathrm{M}$ and $15 \mu \mathrm{M}$. The results are shown in Figure 2 .
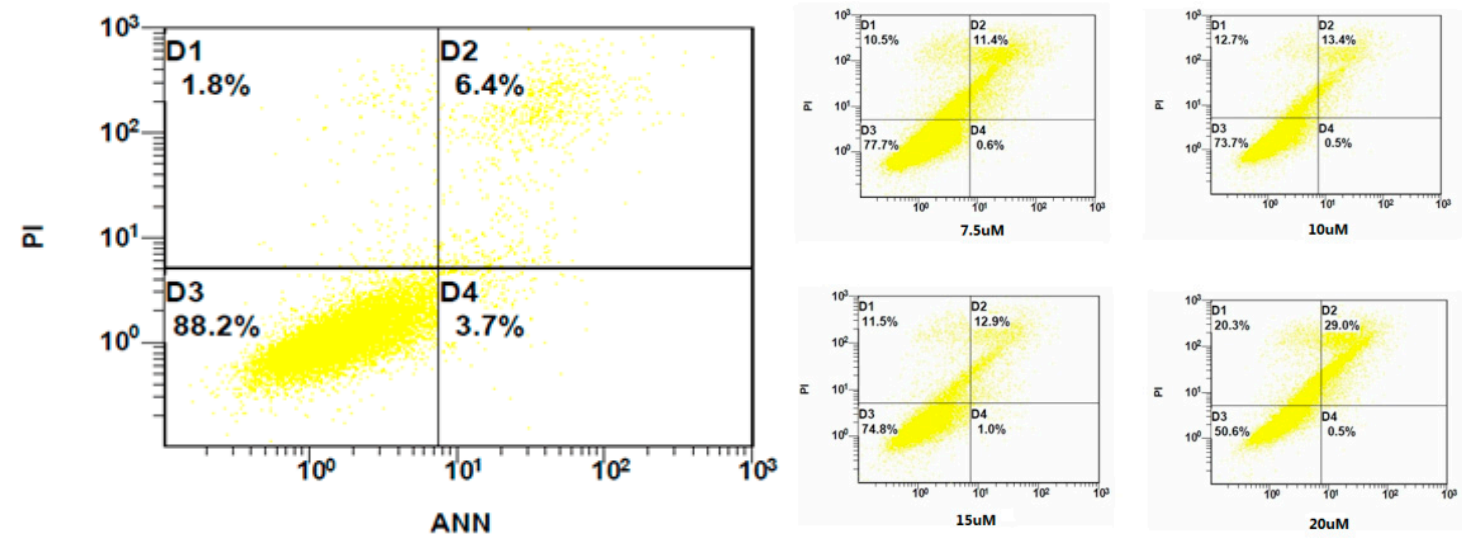

Figure 2. Flow cytometry results. 
Using the Pharmacophores Ligand module of the Profiler DS3.1 program to reverse the drug group screening, a total of 1300 targets were screened. According to the reverse pharmacodynamic docking method, $\mathbf{5 b}$ has a good effect on CDK2. The results are shown in Table 2 . In the molecular docking of compound $\mathbf{5 b}$ and CDK2, the oxygen atom of the sulfonic group and SER141 amino acid residues form hydrogen bond interactions, suggesting that compound $\mathbf{5 b}$ has a good binding capacity to CDK2 protein. The results are shown in Figure 3.

Table 2. Reverse pharmacodynamic docking results.

\begin{tabular}{ccccccc}
\hline \multicolumn{7}{c}{ Pharmacophores that Fit Each Molecule } \\
\hline Name & Pharmacophore & Fit Value & Class & Subclass & Family & Acronym \\
\hline 5b & 1e9h-inr-2.50-h-1 & 0.036708 & Enzymes & $\begin{array}{c}\text { EC2. } \\
\text {-(transferases) }\end{array}$ & $\begin{array}{c}\text { Kinases } \\
\text { (serine threonine) }\end{array}$ & CDK2 \\
\hline
\end{tabular}

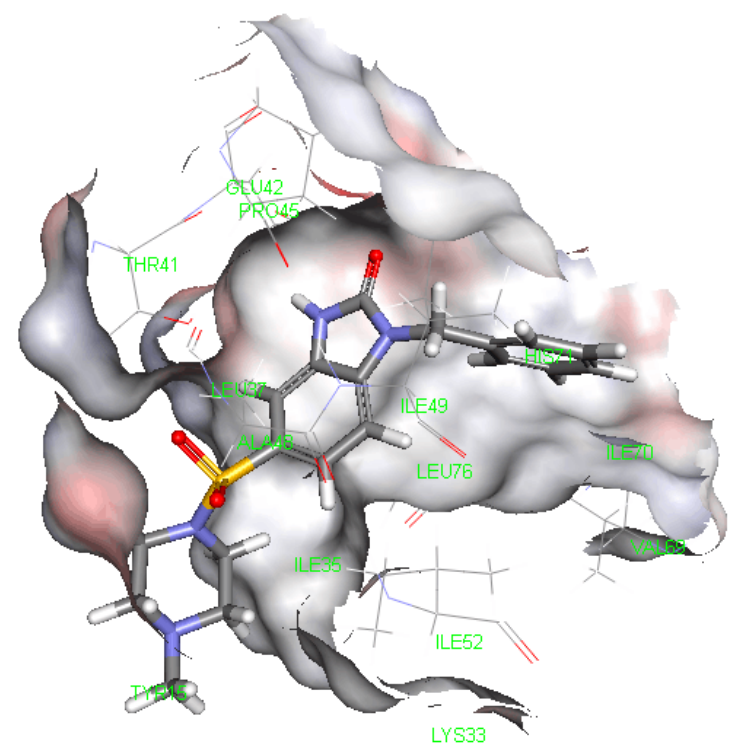

Figure 3. Molecular docking of $5 \mathbf{b}$ and CDK2.

\subsection{Discussion}

We have designed and synthesized some novel 5-hydrosulfonyl-1H-benzo[d]imidazol-2(3H)-one derivatives, and evaluated their antitumor activities against the A549, HCC1937 and MDA-MB-468 cell lines. Many of the new compounds displayed cytotoxicity toward the tested cell lines. The structure-activity relationship results suggest that sulfamine groups $(R)$ and substituted phenyl or benzyl $\left(R_{1}\right)$ substituent groups in the dihydrobenzoimidazol-2-one ring are associated with the cytotoxicity activity. When $R_{1}$ is phenyl and $R$ is 1-methylpiperazine (compound $5 a$ ) or tetrahydro-2H-pyran (compound $5 \mathbf{b}$ ), the activity toward the A549, HCC1937 and MDA-MB-468 cell lines is good ( $\mathrm{IC}_{50}=2.6-9 \mu \mathrm{M}$ ), whereas when $\mathrm{R}$ is cyclohexanamine (compound $5 \mathrm{c}$ ) or piperidine (compound 5d) the resulting compound exhibits equivalent to 10-fold inferior potency compared to $5 \mathbf{a}$ and $5 \mathbf{b}$. On the other hand, the aliphatic moieties $5 \mathbf{h}$ and $5 \mathbf{i}$ and the various amino moieties found in $\mathbf{5 e}, \mathbf{5 f}, \mathbf{5} \mathbf{g}$ and $\mathbf{5 j}$ resulted in almost no activity. When $\mathrm{R}_{1}$ is benzyl, various $\mathrm{R}$ moieties exhibited similar activity as when $R_{1}$ was phenyl. Further studies on additional related structural modifications are currently in progress in our laboratory. To investigate the apoptotic effects in HCC1937 cells following stimulation with $7.5,10,15$ or $20 \mu \mathrm{mol} / \mathrm{L}$ of $\mathbf{5 b}$, flow cytometry analyses were used. The data revealed that the $5 \mathbf{b}$-induced apoptosis rate of HCC1937 cells increased from $11.4 \%$ to $29 \%$ as the concentration increased. 


\section{Materials and Methods}

\subsection{General Information}

Chemical reagents were obtained from commercial suppliers, and were dried and purified by standard methods when necessary. The progress of reactions was monitored by thin layer chromatography (TLC) using silica gel plates. The ${ }^{1} \mathrm{H}-\mathrm{NMR}$ and ${ }^{13} \mathrm{C}-\mathrm{NMR}$ spectra were recorded on a Bruker (Bruker, Rheinstetten, Germany) AVANCE III400 Hz spectrometer using $\mathrm{CDCl}_{3}$ or DMSO- $d_{6}$ as solvent. Tetramethylsilane $(\delta=0.00 \mathrm{ppm})$ was used as an internal standard. HRMS data were obtained using Bruker micrOTOF-Q instrument or TOF-MS instrument.

\subsection{Synthesis}

The preparation of the compounds $\mathbf{5 a - 0}$ was accomplished by the series of steps described below for the preparation of $\mathbf{5 a}$.

\subsubsection{N-Benzyl-2-Nitroaniline (2a)}

To a solution of 2-nitroaniline $(\mathbf{1}, 1.38 \mathrm{~g}, 10 \mathrm{mmol})$ and $\mathrm{NaOH}(480 \mathrm{mg}, 12 \mathrm{mmol})$ in acetone $(20 \mathrm{~mL})$, the reaction mixture was maintained at $65^{\circ} \mathrm{C}$ over $15 \mathrm{~min}$ while stirring, then benzyl bromide $(1.43 \mathrm{~mL}, 12 \mathrm{mmol})$ was added dropwise over $5 \mathrm{~min}$. After the suspension was stirred at $65{ }^{\circ} \mathrm{C}$ for $1 \mathrm{~h}$, the reaction mixture was poured into water and extracted with ethyl acetate $(3 \times 15 \mathrm{~mL})$. The combined organic layers were washed by brine $(3 \times 15 \mathrm{~mL})$ and dried over anhydrous $\mathrm{MgSO}_{4}$. The solvent was removed in vacuo, and the residue was purified by flash chromatography on silica gel to obtain $2 \mathrm{a}(2.1 \mathrm{~g}, 92 \%)$ as a yellow solid. ${ }^{1} \mathrm{H}-\mathrm{NMR}\left(\mathrm{DMSO}-d_{6}\right) \delta \mathrm{ppm}: 8.66(\mathrm{~s}, 1 \mathrm{H}, \mathrm{NH}), 8.08(\mathrm{~d}, 1 \mathrm{H}$, ArH), $7.45(\mathrm{t}, 1 \mathrm{H}, \mathrm{ArH}), 7.39-7.33(\mathrm{~m}, 4 \mathrm{H}, \mathrm{ArH}), 7.25(\mathrm{t}, 1 \mathrm{H}, \mathrm{ArH}), 6.92(\mathrm{~d}, 1 \mathrm{H}, \mathrm{ArH}), 6.67(\mathrm{t}, 1 \mathrm{H}, \mathrm{ArH})$ $4.63\left(\mathrm{~d}, 2 \mathrm{H}, \mathrm{CH}_{2}\right) .{ }^{13} \mathrm{C}-\mathrm{NMR}$ (DMSO- $\left.d_{6}\right) \delta$ ppm: 144.89, 138.49, 136.37, 131.29, 128.55, 128.55, 127.04, $126.91,126.91,126.19,115.39,114.88,45.68$. HRMS: calcd. for $\mathrm{C}_{13} \mathrm{H}_{12} \mathrm{~N}_{2} \mathrm{O}_{2}{ }^{+}[\mathrm{M}+\mathrm{Na}]^{+}: 251.0796$, found: 251.0798. Melting point: $73.8-74.9{ }^{\circ} \mathrm{C} .{ }^{1} \mathrm{H}-\mathrm{NMR},{ }^{13} \mathrm{C}-\mathrm{NMR}$ and HRMS spectra are provided in the Supplementary Materials.

\subsubsection{N-Benzyl-4-(morpholinosulfonyl)-2-nitroaniline (3a)}

Chlorosulfonic acid $(10 \mathrm{~mL})$ was cooled at $0{ }^{\circ} \mathrm{C}$ for $10 \mathrm{~min}$, then the compound $2 \mathrm{a}(2.28 \mathrm{~g}, 10 \mathrm{mmol})$ was added slowly. After stirring for $4 \mathrm{~h}$ at $0{ }^{\circ} \mathrm{C}$, the reaction mixture was poured into ice water $(50 \mathrm{~mL})$, and then extracted with ethyl acetate $(3 \times 15 \mathrm{~mL})$. The organic layer was dried over anhydrous $\mathrm{MgSO}_{4}$ and concentrated in vacuo. The residue was purified by flash chromatography on silica gel to obtain the intermediate. A solution of the intermediate $(327 \mathrm{mg}, 1 \mathrm{mmol})$ and morpholine $(87 \mu \mathrm{L}, 1 \mathrm{mmol})$ in tetrahydrofuran $(5 \mathrm{~mL})$ was stirred for $0.5 \mathrm{~h}$ at room temperature, then the solvent was removed in vacuo. The residual solid was purified by flash chromatography on silica gel to give compound 3a $(358 \mathrm{mg}, 65 \%)$ as a yellow solid. Melting point: $80.1-81.7{ }^{\circ} \mathrm{C} .{ }^{1} \mathrm{H}-\mathrm{NMR}\left(\mathrm{DMSO}-d_{6}\right) \delta \mathrm{ppm}: 8.78(\mathrm{t}, 1 \mathrm{H}$, $\mathrm{NH}), 8.09$ (d, 1H, ArH), 7.77-7.60 (m, 4H, ArH), 7.43 (t, 1H, ArH), 6.90 (d, 1H, ArH), 6.69 (t, 1H, ArH), $4.78\left(\mathrm{~d}, 2 \mathrm{H}, \mathrm{CH}_{2}\right), 3.57$ (s, 4H, $\left.\mathrm{CH}_{2}\right), 2.75$ (s, 4H, $\left.\mathrm{CH}_{2}\right) .{ }^{13} \mathrm{C}-\mathrm{NMR}$ (DMSO-d 6 ) $\delta$ ppm: 144.47, 140.61, 136.27, 134.46, 131.98, 131.98, 131.64, 131.64, 129.68, 126.27, 125.86, 114.96, 65.18, 65.18, 45.77, 45.77, 45.02. HRMS: calcd. for $\mathrm{C}_{17} \mathrm{H}_{19} \mathrm{~N}_{3} \mathrm{O}_{5} \mathrm{~S}^{+}[\mathrm{M}+\mathrm{Na}]^{+}: 400.0943$, found: 400.0946 .

\subsubsection{N'-Benzyl-4-(morpholinosulfonyl)benzene-1,2-diamine (4a)}

A mixture of silica gel (1.5 g, 4 equivalents), and iron dust (140 mg, $2.5 \mathrm{mmol})$ was added to an ethyl alcohol/water (4:1) mixture $(10 \mathrm{~mL})$, the mixture was adjusted to $\mathrm{pH} 4 \sim 5 \mathrm{with} 1 \mathrm{~N} \mathrm{HCl}$. After stirring for $10 \mathrm{~min}$ at $80^{\circ} \mathrm{C}$, compound $3 \mathrm{a}(377 \mathrm{mg}, 1 \mathrm{mmol})$ was added. The reaction mixture was stirred for $1 \mathrm{~h}$ at the same temperature and filtered, the filtrate was adjusted to $\mathrm{pH}$ 8 9 with saturated $\mathrm{NaHCO}_{3}$, some silica gel was added to the mixture and filtered. The mixture was added to the water $(100 \mathrm{~mL})$, and then extracted with ethyl acetate $(3 \times 15 \mathrm{~mL})$. The organic layer was dried 
over anhydrous $\mathrm{MgSO}_{4}$ and concentrated in vacuo. The residue was purified by flash chromatography on silica gel to obtain $4 \mathrm{a}(277 \mathrm{mg}, 80 \%)$ as a brown solid. Melting point: $178.3-179.4{ }^{\circ} \mathrm{C}$. ${ }^{1} \mathrm{H}-\mathrm{NMR}$ (DMSO- $\left.d_{6}\right) \delta$ ppm: $7.73(\mathrm{~d}, 1 \mathrm{H}, \mathrm{ArH}), 7.65-7.54(\mathrm{~m}, 3 \mathrm{H}, \mathrm{ArH}), 6.55(\mathrm{~d}, 1 \mathrm{H}, \mathrm{ArH}), 6.35-6.34(\mathrm{~m}, 2 \mathrm{H}$, $\mathrm{ArH}), 6.26(\mathrm{~d}, 1 \mathrm{H}, \mathrm{ArH}), 5.35(\mathrm{t}, 1 \mathrm{H}, \mathrm{NH}), 4.58\left(\mathrm{~s}, 2 \mathrm{H}, \mathrm{NH}_{2}\right), 4.44\left(\mathrm{~d}, 2 \mathrm{H}, \mathrm{CH}_{2}\right), 3.55\left(\mathrm{t}, 4 \mathrm{H}, \mathrm{CH}_{2}\right)$, $2.68\left(\mathrm{~s}, 4 \mathrm{H}, \mathrm{CH}_{2}\right) .{ }^{13} \mathrm{C}-\mathrm{NMR}$ (DMSO- $\left.d_{6}\right) \delta$ ppm: 142.41, 135.46, 134.77, 134.07, 132.21, 129.25, 129.25, $126.02,126.02,125.70,117.23,114.23,65.19,65.19,46.15,45.74,45.74$. HRMS: calcd. for $\mathrm{C}_{17} \mathrm{H}_{21} \mathrm{~N}_{3} \mathrm{O}_{3} \mathrm{~S}^{+}$ $[\mathrm{M}+\mathrm{H}]^{+}: 348.1382$, found:348.1368.

\subsubsection{Benzyl-5-(morpholinosulfonyl)-1H-benzo[d]imidazol-2(3H)-one (5a)}

To a solution of $4 \mathbf{a}(347 \mathrm{mg}, 1 \mathrm{mmol})$ in anhydrous tetrahydrofuran $(3 \mathrm{~mL})$ at $0{ }^{\circ} \mathrm{C}$ for $10 \mathrm{~min}$, a solution of triphosgene $(148 \mathrm{mg}, 0.6 \mathrm{mmol})$ in anhydrous tetrahydrofuran $(3 \mathrm{~mL})$ was added dropwise at the same temperature. After stirring for $30 \mathrm{~min}$, the reaction mixture was poured into water $(30 \mathrm{~mL})$ and adjusted to $\mathrm{pH} 8 \sim 9$ with saturated $\mathrm{NaHCO}_{3}$, and then extracted with ethyl acetate $(3 \times 15 \mathrm{~mL})$. The organic layer was dried over anhydrous $\mathrm{MgSO}_{4}$ and concentrated in vacuo. The residue was purified by flash chromatography on silica gel to afford $\mathbf{5 a}(280 \mathrm{mg}, 75 \%)$ as a white solid. Melting point: $198.5-200.1{ }^{\circ} \mathrm{C} .{ }^{1} \mathrm{H}-\mathrm{NMR}$ (DMSO-d $\left.{ }_{6}\right) \delta$ ppm: $11.02(\mathrm{~s}, 1 \mathrm{H}, \mathrm{NH}), 7.65(\mathrm{~d}, 4 \mathrm{H}, \mathrm{ArH}), 7.08-6.93(\mathrm{~m}$, $4 \mathrm{H}, \mathrm{ArH}), 5.16\left(\mathrm{~s}, 2 \mathrm{H}, \mathrm{CH}_{2}\right), 3.57\left(\mathrm{~s}, 4 \mathrm{H}, \mathrm{CH}_{2}\right), 2.76\left(\mathrm{~s}, 4 \mathrm{H}, \mathrm{CH}_{2}\right) .{ }^{13} \mathrm{C}-\mathrm{NMR}\left(\mathrm{DMSO}-d_{6}\right) \delta \mathrm{ppm}: 154.33$, $138.89,134.64,132.20,129.89,128.29,128.29,126.59,126.59,126.24,121.25,120.59,108.98,65.17,65.17$, 45.75, 42.67, 42.67. HRMS: calcd. for $\mathrm{C}_{18} \mathrm{H}_{19} \mathrm{~N}_{3} \mathrm{O}_{4} \mathrm{~S}^{+}[\mathrm{M}+\mathrm{Na}]^{+}$: 396.0994, found: 396.0992 .

The following compounds were similarly prepared:

Benzyl-5-(4-methylpiperazin-1-ylsulfonyl)-1H-benzo[d]imidazol-2(3H)-one (5b). Melting point: $107.2-108.1^{\circ} \mathrm{C}$. ${ }^{1} \mathrm{H}-\mathrm{NMR}$ (DMSO- $\left.d_{6}\right) \delta$ ppm: $11.01(\mathrm{~s}, 1 \mathrm{H}, \mathrm{NH}), 7.64(\mathrm{~d}, 4 \mathrm{H}, \mathrm{ArH}), 7.08-6.93(\mathrm{~m}, 4 \mathrm{H}, \mathrm{ArH}), 5.15(\mathrm{~s}, 2 \mathrm{H}$, $\left.\mathrm{CH}_{2}\right), 2.79\left(\mathrm{~s}, 4 \mathrm{H}, \mathrm{CH}_{2}\right), 2.27\left(\mathrm{~s}, 4 \mathrm{H}, \mathrm{CH}_{2}\right), 2.10\left(\mathrm{~s}, 3 \mathrm{H}, \mathrm{CH}_{3}\right) .{ }^{13} \mathrm{C}-\mathrm{NMR}$ (DMSO- $\left.d_{6}\right) \delta$ ppm: 154.32, 138.84, 135.24, 132.03, 129.78, 128.28, 128.28, 126.46, 126.46, 126.17, 121.22, 120.58, 108.95, 53.33, 53.33, 45.57, 45.57, 45.20, 42.66. HRMS: calcd. for $\mathrm{C}_{19} \mathrm{H}_{22} \mathrm{~N}_{4} \mathrm{O}_{3} \mathrm{~S}^{+}[\mathrm{M}+\mathrm{H}]^{+}: 387.1491$, found: 387.1456.

Benzyl-N-cyclohexyl-2-oxo-2,3-dihydro-1H-benzo[d]imidazole-5-sulfonamide (5c). Melting point: $166.7-167.2{ }^{\circ} \mathrm{C}$. ${ }^{1} \mathrm{H}-\mathrm{NMR}\left(\mathrm{DMSO}-d_{6}\right) \delta$ ppm: $11.01(\mathrm{~d}, 1 \mathrm{H}, \mathrm{NH}), 7.78-7.46(\mathrm{~m}, 5 \mathrm{H}, \mathrm{ArH}, \mathrm{NH}), 6.91-6.98(\mathrm{~m}, 4 \mathrm{H}$, $\mathrm{ArH}), 5.11\left(\mathrm{~s}, 2 \mathrm{H}, \mathrm{CH}_{2}\right), 2.82(\mathrm{~s}, 1 \mathrm{H}, \mathrm{CH}), 1.49-1.41\left(\mathrm{~m}, 5 \mathrm{H}, \mathrm{CH}_{2}\right), 1.09-1.00\left(\mathrm{~m}, 5 \mathrm{H}, \mathrm{CH}_{2}\right) .{ }^{13} \mathrm{C}-\mathrm{NMR}$ (DMSO- $d_{6}$ ) $\delta$ ppm: 154.29, 142.62, 138.43, 131.13, 129.71, 128.31, 128.31, 126.61, 126.61, 125.28, 121.12, $120.51,108.89,52.04,52.04,42.82,33.10,33.10,24.76,24.28$. HRMS: calcd. for $\mathrm{C}_{20} \mathrm{H}_{23} \mathrm{~N}_{3} \mathrm{O}_{3} \mathrm{~S}^{+}[\mathrm{M}+\mathrm{Na}]^{+}$: 408.1358, found: 408.1363 .

Benzyl-5-(piperidin-1-ylsulfonyl)-1H-benzo[d]imidazol-2(3H)-one (5d). Melting point: $189.4-190.5{ }^{\circ} \mathrm{C}$. ${ }^{1} \mathrm{H}-\mathrm{NMR}\left(\mathrm{CDCl}_{3}\right) \delta$ ppm: $9.86(\mathrm{~s}, 1 \mathrm{H}, \mathrm{NH}), 7.69(\mathrm{t}, 2 \mathrm{H}, \mathrm{ArH}), 7.55-7.47(\mathrm{~m}, 2 \mathrm{H}, \mathrm{ArH}), 7.14-6.98(\mathrm{~m}, 3 \mathrm{H}$, $\mathrm{ArH}), 6.81(\mathrm{~d}, 1 \mathrm{H}, \mathrm{ArH}), 5.18\left(\mathrm{~s}, 2 \mathrm{H}, \mathrm{CH}_{2}\right), 2.91\left(\mathrm{t}, 4 \mathrm{H}, \mathrm{CH}_{2}\right), 1.71\left(\mathrm{~s}, 2 \mathrm{H}, \mathrm{CH}_{2}\right), 1.59-1.56\left(\mathrm{~m}, 4 \mathrm{H}, \mathrm{CH}_{2}\right)$. ${ }^{13} \mathrm{C}-\mathrm{NMR}\left(\mathrm{CDCl}_{3}\right) \delta$ ppm: 155.53, 137.55, 137.05, 131.38, 129.63, 127.96, 127.96, 127.00, 127.00, 126.30, $122.12,121.55,109.90,46.87,46.87,44.04,25.12,25.12,23.43$. HRMS: calcd. for $\mathrm{C}_{19} \mathrm{H}_{21} \mathrm{~N}_{3} \mathrm{O}_{3} \mathrm{~S}^{+}[\mathrm{M}+\mathrm{H}]^{+}$: 372.1382, found: 372.1400 .

N,1-Dibenzyl-2-oxo-2,3-dihydro-1H-benzo[d]imidazole-5-sulfonamide (5e). Melting point: $160.6-185.9{ }^{\circ} \mathrm{C}$. ${ }^{1} \mathrm{H}-\mathrm{NMR}$ (DMSO- $\left.d_{6}\right) \delta$ ppm: $11.00(\mathrm{~s}, 1 \mathrm{H}, \mathrm{NH}), 8.16(\mathrm{t}, 1 \mathrm{H}, \mathrm{ArH}), 7.75(\mathrm{~s}, 1 \mathrm{H}, \mathrm{ArH}), 7.67(\mathrm{~d}, 1 \mathrm{H}, \mathrm{ArH})$, 7.56-7.50 (m, 2H, ArH), 7.24-7.14 (m, 4H, ArH), 7.05-6.94 (m, 4H, ArH), 5.09 (s, 2H, $\left.\mathrm{CH}_{2}\right), 3.92(\mathrm{~d}$, $\left.2 \mathrm{H}, \mathrm{CH}_{2}\right), 3.17$ (d, $\left.1 \mathrm{H}, \mathrm{NH}\right) .{ }^{13} \mathrm{C}-\mathrm{NMR}$ (DMSO- $\left.d_{6}\right) \delta \mathrm{ppm}$ : 154.30, 141.11, 138.47, 137.42, 131.16, 129.80, 128.32, 128.32, 128.14, 128.14, 127.50, 127.50, 127.07, 127.07, 125.46, 125.46, 125.17, 121.18, 120.60, 46.09, 42.81. HRMS: calcd. for $\mathrm{C}_{21} \mathrm{H}_{19} \mathrm{~N}_{3} \mathrm{O}_{3} \mathrm{~S}^{+}[\mathrm{M}+\mathrm{Na}]^{+}$: 416.1045, found: 416.1030 .

N-(3-Benzoylphenyl)-1-benzyl-2-oxo-2,3-dihydro-1H-benzo[d]imidazole-5-sulfonamide (5f). Melting point: 85.2-86.3 ${ }^{\circ} \mathrm{C} .{ }^{1} \mathrm{H}-\mathrm{NMR}$ (DMSO- $\left.d_{6}\right) \delta \mathrm{ppm}: 11.00(\mathrm{~d}, 1 \mathrm{H}, \mathrm{NH}), 10.00(\mathrm{~s}, 1 \mathrm{H}, \mathrm{NH}), 7.64-6.89(\mathrm{~m}, 17 \mathrm{H}$, $\mathrm{ArH}), 5.00\left(\mathrm{~d}, 2 \mathrm{H}, \mathrm{CH}_{2}\right) .{ }^{13} \mathrm{C}-\mathrm{NMR}\left(\mathrm{CDCl}_{3}\right) \delta \mathrm{ppm}: 198.58,155.08,139.47,138.78,137.64,137.56,133.84$, $133.21,132.80,131.87,129.78,128.26,128.26,128.21,127.83,127.66,126.60,126.60,126.18,126.18,125.96$, 
123.54, 122.70, 122.07, 121.79, 109.64, 43.79. HRMS: calcd. for $\mathrm{C}_{27} \mathrm{H}_{21} \mathrm{~N}_{3} \mathrm{O}_{4} \mathrm{~S}^{+}[\mathrm{M}+\mathrm{Na}]^{+}: 506.1150$, found: 506.1171 .

Benzyl-2-oxo-N-phenyl-2,3-dihydro-1H-benzo[d]imidazole-5-sulfonamide (5g). Melting point: $91.9-93.3{ }^{\circ} \mathrm{C}$. ${ }^{1} \mathrm{H}-\mathrm{NMR}\left(\mathrm{DMSO}-d_{6}\right) \delta \mathrm{ppm}: 10.98(\mathrm{~s}, 1 \mathrm{H}, \mathrm{NH}), 10.27(\mathrm{~s}, 1 \mathrm{H}, \mathrm{NH}), 7.72-7.40(\mathrm{~m}, 4 \mathrm{H}, \mathrm{ArH}), 7.22-6.88(\mathrm{~m}$, 9H, ArH), $5.04\left(\mathrm{~s}, 2 \mathrm{H}, \mathrm{CH}_{2}\right) .{ }^{13} \mathrm{C}-\mathrm{NMR}$ (DMSO- $\left.d_{6}\right) \delta$ ppm: $154.23,139.86,138.60,137.39,131.83,129.69$, $129.69,129.58,128.99,128.99,125.66,125.66,125.38,124.08,121.14,120.17,119.95,119.95,108.89,54.87$. HRMS: calcd. for $\mathrm{C}_{20} \mathrm{H}_{17} \mathrm{~N}_{3} \mathrm{O}_{3} \mathrm{~S}^{+}[\mathrm{M}+\mathrm{Na}]^{+}:$402.0888, found: 402.0893 .

Benzyl-N-cyclopropyl-2-oxo-2,3-dihydro-1H-benzo[d] imidazole-5-sulfonamide (5h). Melting point: $168.6-170.3^{\circ} \mathrm{C}$. ${ }^{1} \mathrm{H}-\mathrm{NMR}\left(\mathrm{DMSO}-d_{6}\right) \delta \mathrm{ppm}: 11.03(\mathrm{~s}, 1 \mathrm{H}, \mathrm{NH}), 7.90(\mathrm{~s}, 1 \mathrm{H}, \mathrm{NH}), 7.71(\mathrm{~s}, 2 \mathrm{H}, \mathrm{ArH}), 7.61-7.57(\mathrm{~m}$, 2H, ArH), 7.03-6.92 (m, 4H, ArH), $5.13\left(\mathrm{~s}, 2 \mathrm{H}, \mathrm{CH}_{2}\right), 1.99(\mathrm{~s}, 1 \mathrm{H}, \mathrm{CH}), 0.36-0.25\left(\mathrm{~m}, 4 \mathrm{H}, \mathrm{CH}_{2}\right)$. ${ }^{13} \mathrm{C}-\mathrm{NMR}$ (DMSO- $d_{6}$ ) $\delta$ ppm: 154.29, 140.58, 138.44, 131.40, 129.73, 129.55, 128.29, 125.66, 125.31, $121.18,120.55,108.92,107.99,42.77,24.04,4.99,4.99$. HRMS: calcd. for $\mathrm{C}_{17} \mathrm{H}_{17} \mathrm{~N}_{3} \mathrm{O}_{3} \mathrm{~S}^{+}[\mathrm{M}+\mathrm{Na}]^{+}$: 366.0888, found: 366.0860 .

Benzyl-N-isopropyl-2-oxo-2,3-dihydro-1H-benzo[d]imidazole-5-sulfonamide (5i). Melting point: $173.4-174.7^{\circ} \mathrm{C}$. ${ }^{1} \mathrm{H}-\mathrm{NMR}\left(\mathrm{DMSO}-d_{6}\right) \delta$ ppm: $11.02(\mathrm{~s}, 1 \mathrm{H}, \mathrm{NH}), 7.75-7.54(\mathrm{~m}, 5 \mathrm{H}, \mathrm{ArH}), 7.00-6.92(\mathrm{~m}, 4 \mathrm{H}, \mathrm{ArH}), 5.12$ $\left(\mathrm{s}, 2 \mathrm{H}, \mathrm{CH}_{2}\right), 3.20-3.14(\mathrm{~m}, 1 \mathrm{H}, \mathrm{NH}), 0.87\left(\mathrm{~d}, 6 \mathrm{H}, \mathrm{CH}_{3}\right) .{ }^{13} \mathrm{C}-\mathrm{NMR}$ (DMSO- $\left.d_{6}\right) \delta \mathrm{ppm}: 154.30,142.22$, 138.47, 131.08, 129.73, 129.50, 129.50, 128.31, 128.31, 125.37, 124.95, 121.14, 120.54, 45.20, 41.28, 23.05, 23.05. HRMS: calcd. for $\mathrm{C}_{17} \mathrm{H}_{19} \mathrm{~N}_{3} \mathrm{O}_{3} \mathrm{~S}^{+}[\mathrm{M}+\mathrm{Na}]^{+}$: 368.1045, found: 368.1051 .

Benzyl-2-oxo-N-o-tolyl-2,3-dihydro-1H-benzo[d]imidazole-5-sulfonamide (5j). Melting point: $223.6-225.7^{\circ} \mathrm{C}$. ${ }^{1} \mathrm{H}-\mathrm{NMR}\left(\mathrm{DMSO}-d_{6}\right) \delta \mathrm{ppm}: 10.97(\mathrm{~s}, 1 \mathrm{H}, \mathrm{NH}), 9.55(\mathrm{~d}, 1 \mathrm{H}, \mathrm{NH}), 7.62-7.42(\mathrm{~m}, 4 \mathrm{H}, \mathrm{ArH}), 7.09-6.85$ $(\mathrm{m}, 8 \mathrm{H}, \mathrm{ArH}), 5.04\left(\mathrm{~s}, 2 \mathrm{H}, \mathrm{CH}_{2}\right), 1.83\left(\mathrm{~s}, 3 \mathrm{H}, \mathrm{CH}_{3}\right) .{ }^{13} \mathrm{C}-\mathrm{NMR}$ (DMSO- $\left.d_{6}\right) \delta \mathrm{ppm:} \mathrm{154.22,} \mathrm{140.94,} \mathrm{138.60,}$ 134.47, 134.08, 131.73, 130.55, 129.71, 129.46, 128.31, 128.31, 126.41, 126.41, 126.15, 125.63, 125.15, 121.14, 120.55, 108.89, 42.75, 17.38. HRMS: calcd. for $\mathrm{C}_{21} \mathrm{H}_{19} \mathrm{~N}_{3} \mathrm{O}_{3} \mathrm{~S}^{+}[\mathrm{M}+\mathrm{Na}]^{+}: 416.1045$, found: 416.1067.

N-Cyclohexyl-2-oxo-1-phenethyl-2,3-dihydro-1H-benzo[d]imidazole-5-sulfonamide (5k). Melting point: 187.0-188.5 ${ }^{\circ} \mathrm{C} .{ }^{1} \mathrm{H}-\mathrm{NMR}$ (DMSO-d $\left.d_{6}\right) \delta 10.80$ (s, 1H, NH), 7.68 (d, 2H, ArH), $7.56(\mathrm{~d}, 1 \mathrm{H}, \mathrm{ArH})$, $7.43(\mathrm{t}, 2 \mathrm{H}, \mathrm{ArH}), 7.06(\mathrm{~s}, 1 \mathrm{H}, \mathrm{ArH}), 6.95(\mathrm{~s}, 3 \mathrm{H}, \mathrm{ArH}), 4.03\left(\mathrm{dd}, 2 \mathrm{H}, \mathrm{CH}_{2}\right), 3.04\left(\mathrm{~s}, 2 \mathrm{H}, \mathrm{CH}_{2}\right), 2.87(\mathrm{~s}, 1 \mathrm{H}$, $\mathrm{CH}), 1.99(\mathrm{~s}, 1 \mathrm{H}, \mathrm{NH}), 1.56-1.39\left(\mathrm{~m}, 5 \mathrm{H}, \mathrm{CH}_{2}\right), 1.23-1.08\left(\mathrm{~m}, 5 \mathrm{H}, \mathrm{CH}_{2}\right) .{ }^{13} \mathrm{C}-\mathrm{NMR}$ (DMSO-d 6 ) $\delta$ ppm: $153.99,142.98,140.29,129.98,129.98,129.46,129.46,128.08,126.25,120.67,120.33,108.62,107.72,59.72$, 51.94, 33.52, 33.13, 33.13, 26.30, 24.83, 24.83. HRMS: calcd. for $\mathrm{C}_{21} \mathrm{H}_{19} \mathrm{~N}_{3} \mathrm{O}_{3} \mathrm{~S}^{+}[\mathrm{M}+\mathrm{Na}]^{+}: 422.1514$, found: 422.1471 .

5-(4-Methylpiperazin-1-ylsulfonyl)-1-phenethyl-1H-benzo[d]imidazol-2(3H)-one (51). Melting point: $218.2-219.6{ }^{\circ} \mathrm{C}$. ${ }^{1} \mathrm{H}-\mathrm{NMR}\left(\mathrm{DMSO}-d_{6}\right) \delta$ ppm: $10.81(\mathrm{~s}, 1 \mathrm{H}, \mathrm{NH}), 7.57$ (d, 2H, ArH), $7.46(\mathrm{~d}, 2 \mathrm{H}, \mathrm{ArH}), 6.93(\mathrm{~s}, 4 \mathrm{H}, \mathrm{ArH})$, $4.06\left(\mathrm{t}, 2 \mathrm{H}, \mathrm{CH}_{2}\right), 3.07\left(\mathrm{t}, 2 \mathrm{H}, \mathrm{CH}_{2}\right), 2.80\left(\mathrm{~s}, 4 \mathrm{H}, \mathrm{CH}_{2}\right), 2.34\left(\mathrm{~s}, 4 \mathrm{H}, \mathrm{CH}_{2}\right), 2.15\left(\mathrm{~s}, 3 \mathrm{H}, \mathrm{CH}_{3}\right) .{ }^{13} \mathrm{C}-\mathrm{NMR}$ (DMSO- $d_{6}$ ) $\delta$ ppm: 153.99, 144.44, 132.62, 130.02, 129.80, 127.97, 127.97, 127.56, 127.56, 120.65, 120.49, $108.63,107.62,53.45,53.45,45.61,45.61,45.26,40.95,33.72$. HRMS: calcd. for $\mathrm{C}_{20} \mathrm{H}_{24} \mathrm{~N}_{4} \mathrm{O}_{3} \mathrm{~S}^{+}[\mathrm{M}+\mathrm{Na}]^{+}$: 423.1467, found: 423.1453 .

N-Cyclopropyl-2-oxo-1-phenethyl-2,3-dihydro-1H-benzo[d]imidazole-5-sulfonamide (5m). Melting point: 217.8-218.9 ${ }^{\circ} \mathrm{C} .{ }^{1} \mathrm{H}-\mathrm{NMR}\left(\mathrm{DMSO}-d_{6}\right) \delta \mathrm{ppm}: 10.80(\mathrm{~s}, 1 \mathrm{H}, \mathrm{NH}), 7.82(\mathrm{~s}, 1 \mathrm{H}, \mathrm{NH}), 7.67(\mathrm{~d}, 2 \mathrm{H}, \mathrm{ArH})$, $7.44(\mathrm{~d}, 2 \mathrm{H}, \mathrm{ArH}), 7.03(\mathrm{~s}, 1 \mathrm{H}, \mathrm{ArH}), 6.93(\mathrm{~s}, 3 \mathrm{H}, \mathrm{ArH}), 4.07\left(\mathrm{t}, 2 \mathrm{H}, \mathrm{CH}_{2}\right), 3.06\left(\mathrm{t}, 2 \mathrm{H}, \mathrm{CH}_{2}\right), 1.96(\mathrm{~s}, 1 \mathrm{H}$, $\mathrm{CH}), 0.44-0.34\left(\mathrm{~m}, 4 \mathrm{H}, \mathrm{CH}_{2}\right) .{ }^{13} \mathrm{C}-\mathrm{NMR}$ (DMSO- $\left.d_{6}\right) \delta \mathrm{ppm}: 154.00,143.45,138.19,130.00,129.53,128.05$, $128.05,126.81,126.81,120.64,120.31,108.61,107.70,40.90,33.55,23.97,5.02,5.02$. HRMS: calcd. for $\mathrm{C}_{20} \mathrm{H}_{24} \mathrm{~N}_{4} \mathrm{O}_{3} \mathrm{~S}^{+}[\mathrm{M}+\mathrm{Na}]^{+}:$380.1045, found: 380.1005 .

Phenethyl-5-(piperidin-1-ylsulfonyl)-1H-benzo[d]imidazol-2(3H)-one (5n). Melting point: $183.3-184.3{ }^{\circ} \mathrm{C}$. ${ }^{1} \mathrm{H}-\mathrm{NMR}\left(\mathrm{DMSO}-d_{6}\right) \delta \mathrm{ppm}: 10.81(\mathrm{~s}, 1 \mathrm{H}, \mathrm{NH}), 7.56(\mathrm{~d}, 2 \mathrm{H}, \mathrm{ArH}), 7.44(\mathrm{~d}, 2 \mathrm{H}, \mathrm{ArH}), 6.97-6.90(\mathrm{~m}, 4 \mathrm{H}$, $\mathrm{ArH}), 3.07\left(\mathrm{t}, 2 \mathrm{H}, \mathrm{CH}_{2}\right), 2.75\left(\mathrm{t}, 4 \mathrm{H}, \mathrm{CH}_{2}\right), 1.99\left(\mathrm{~s}, 1 \mathrm{H}, \mathrm{CH}_{2}\right), 1.51\left(\mathrm{~d}, 4 \mathrm{H}, \mathrm{CH}_{2}\right), 1.37\left(\mathrm{t}, 2 \mathrm{H}, \mathrm{CH}_{2}\right), 1.18(\mathrm{t}$, $1 \mathrm{H}, \mathrm{CH}_{2}$ ). ${ }^{13} \mathrm{C}-\mathrm{NMR}$ (DMSO- $d_{6}$ ) $\delta$ ppm: 157.26, 141.15, 138.64, 134.21, 129.19, 129.19, 129.06, 129.06, 
126.36, 123.51, 122.25, 113.41, 108.10, 48.21, 48.21, 45.89, 35.33, 24.74, 24.74, 23.42. HRMS: calcd. for $\mathrm{C}_{20} \mathrm{H}_{23} \mathrm{~N}_{3} \mathrm{O}_{3} \mathrm{~S}^{+}[\mathrm{M}+\mathrm{H}]^{+}: 386.1538$, found: 386.1543 .

5-(Morpholinosulfonyl)-1-phenethyl-1H-benzo[d]imidazol-2(3H)-one (5o). Melting point: $102.3-104.1^{\circ} \mathrm{C}$. ${ }^{1} \mathrm{H}-\mathrm{NMR}$ (DMSO- $d_{6}$ ) $\delta$ ppm: $10.79(\mathrm{~d}, 1 \mathrm{H}, \mathrm{NH}), 7.5-7.45(\mathrm{~m}, 4 \mathrm{H}, \mathrm{ArH}), 6.96(\mathrm{dd}, 4 \mathrm{H}, \mathrm{ArH}), 4.10-4.06(\mathrm{~m}$, 2H, $\left.\mathrm{CH}_{2}\right), 3.60\left(\mathrm{~s}, 4 \mathrm{H}, \mathrm{CH}_{2}\right), 3.14-3.06\left(\mathrm{~m}, 2 \mathrm{H}, \mathrm{CH}_{2}\right), 2.71\left(\mathrm{~d}, 4 \mathrm{H}, \mathrm{CH}_{2}\right) \cdot{ }^{13} \mathrm{C}-\mathrm{NMR}$ (DMSO- $\left.d_{6}\right) \delta \mathrm{ppm}$ : 154.03, 144.66, 140.23, 134.27, 134.03, 132.16, 129.32, 127.62, 125.64, 120.69, 120.31, 108.64, 107.73, 65.21, 65.21, 45.75, 45.75, 40.96, 33.72. HRMS: calcd. for $\mathrm{C}_{19} \mathrm{H}_{21} \mathrm{~N}_{3} \mathrm{O}_{4} \mathrm{~S}^{+}[\mathrm{M}+\mathrm{Na}]^{+}: 410.1150$, found: 410.1155 .

\subsection{Cell Proliferation Assay}

All target compounds were evaluated for their cytotoxic activities (\%) in vitro against human cancer cell lines, including A549, HCC1937 and MDA-MB-468, by applying the MTT colorimetric assay. The cell concentration was adjusted to $2 \times 10^{4} / \mathrm{mL}$ using complete culture medium, then $100 \mu \mathrm{L}$ cell suspension was inoculated in each well of a 96-well culture plate and cultured for $24 \mathrm{~h}$. All the target compounds were adjusted to different concentrations ranging from $1.25 \mu \mathrm{mol} / \mathrm{L}$ to $40 \mu \mathrm{mol} / \mathrm{L}$, then $100 \mu \mathrm{L}$ solution was added to each well of 96-well culture plate and $100 \mu \mathrm{L}$ complete culture medium was added to the control group. Cells were placed under $5 \% \mathrm{CO}_{2}$ at $37^{\circ} \mathrm{C}$ and cultured for $48 \mathrm{~h}$. Then $5 \mathrm{mg} / \mathrm{mL}$ MTT $20 \mu \mathrm{L}$ was added to each well of 96-well culture plate and cultured for another $2 \sim 4 \mathrm{~h}$. The medium was removed and $150 \mu \mathrm{L}$ DMSO was added to each well of 96-well culture plate, then oscillated and mixed $15 \mathrm{~min}$. The absorbance (A) of each well was measured in terms of optical density at a wavelength of $570 \mathrm{~nm}$, each cell viability assay was performed in triplicate and taken the average value, then calculated the $\mathrm{IC}_{50}$. Doxorubicin was the positive control drug.

\subsection{Flow Cytometry}

The Annexin V-FITC apoptosis detection kit was used to detect apoptotic cells according to the manufacturer's protocol. Briefly, cells were gently washed with PBS and collected using trypsinization, disaggregated to a single cell suspension and incubated with $5 \mu \mathrm{L}$ of Annexin V-FITC and $10 \mu \mathrm{L}$ of a PI solution for $15 \mathrm{~min}$ in the dark. The apoptotic cells were detected using a flow cytometer (BD, San Diego, CA, USA), then quantified and the percentage of apoptotic cells measured.

\section{Conclusions}

In conclusion, we have successfully developed an efficient method for the synthesis of a series of novel 5-hydrosulfonyl-1H-benzo[d]imidazol-2(3H)-one derivatives via a simple four step route. This method has the advantages of convenient operation, the ready availability of starting reagents, mild reaction conditions employed, as well as good yields. Next the prepared compounds were screened against A549, HCC1937 and MDA-MB-468 human tumor cell lines using an MTT cell proliferation assay. The results suggested that some of these compounds were effective in inhibiting these cancer cells' growth, especially compound $5 \mathbf{b}$ which was the most potent one $\left(\mathrm{IC}_{50}=2.6 \mu \mathrm{M}\right)$ against HCC1937. The SAR data was summarized. Unfortunately only derivatives where $\mathrm{R}_{1}$ was a benzyl or phenyl group could be prepared and other aromatic and heterocyclic aromatics ccould not be synthesized by us. The flow cytometry data revealed that the $\mathbf{5 b}$ induced apoptosis of HCC1937 cells as the concentration increased.

Supplementary Materials: Supplementary materials can be accessed at: http:/ /www.mdpi.com/1420-3049/21/ 4/516/s1. Characterization data on compounds $\mathbf{2 a}, \mathbf{3 a}, \mathbf{4 a}, \mathbf{5 a}-\mathbf{o}$.

Acknowledgments: We are grateful to Rui Li (Sichuan University) for his MS analysis on this manuscript. Financial support from Natural Science Foundation of China (No. 81302643), China Postdoctoral Science Foundation (No. 2014M552374), and Sichuan Provincial Health Department Research Project (No. 120077) is gratefully acknowledged.

Author Contributions: Guang Ouyang, Rongsheng Tong, Jinqi Li, Lan Bai, Liang Ouyang, Xingmei Duan, Fengqiong Li, Pin He, Jianyou Shi and Yuxin He performed the experiments. Jianyou Shi and Yuxin He designed 
the experiments, Guang Ouyang and Jianyou Shi wrote the paper. All authors took part in data analysis and discussion. All authors read and approved the final manuscript.

Conflicts of Interest: The authors declare no conflict of interest.

\section{References}

1. Goss, P.E.; Strasser-Weipp, K.; Lee-Bychkovskyet, B.L. Challenges to effective cancer control in China, India, and Russia. Lancet. Oncol. 2014, 15, 489-538. [CrossRef]

2. Bray, F.; Jemal, A.; Greyet, N. Global cancer transitions according to the Human Development Index (2008-2030): A population-based study. Lancet. Oncol. 2012, 13, 790-801. [CrossRef]

3. Pettit, G.R.; McNulty, J.; Herald, D.L.; Doubek, D.L.; Chapuis, J.C.; Schmidt, J.M.; Tackett, L.P.; Boyd, M.R. Antineoplastic Agents. 362. Isolation and X-ray crystal structure of dibromophakellstatin from the Indian Ocean Sponge Phakellia Mauritiana. J. Nat. Prod. 1997, 60, 180-183. [CrossRef] [PubMed]

4. Cafieri, F.; Fattorusso, E.; Mangoni, A. Longamide and 3,7-dimethylisoguanine, two novel alkaloids from the marine sponge Agel aslongissima. Tetrahedron Lett. 1995, 36, 7893-7896. [CrossRef]

5. Liu, W.; Lau, F.; Liu, K. Benzimidazolones: A New class of selective peroxisome proliferator-activated receptor $\gamma(\operatorname{PPAR} \gamma)$ modulators. J. Med. Chem. 2011, 54, 8541-8554. [CrossRef] [PubMed]

6. Budzik, B.; Garzya, V.; Shi, D. Selective, novel $N$-substituted benzimidazolones as potent, selective, CNS-penetrant, and orally active M1 mAChR agonists. ACS Med. Chem. Lett. 2010, 1, 244-248. [CrossRef] [PubMed]

7. Li, S.K.; Ji, Z.Q.; Zhang, J.W. Synthesis of 1-Acyl-3-isopropenylbenzimidazolone derivatives and their activity against botrytis cinerea. J. Agric. Food. Chem. 2010, 58, 2668-2672. [CrossRef] [PubMed]

8. Scherrer, N.C.; Stefan, Z.; Francoise, D. Synthetic organic pigments of the 20th and 21st century relevant to artist's paints: Raman spectra reference collection. Spectrochim. Acta A Mol. Biomol. Spectrosc. 2009, 73, 505-524. [CrossRef] [PubMed]

9. Bruncko, M.; Tahir, S.K.; Song, X. N-Aryl-benzimidazolones as novel small molecule HSP90 inhibitors. Bioorg. Med. Chem. Lett. 2010, 20, 7503-7506. [CrossRef] [PubMed]

10. Wu, Z.; Robinson, R.G.; Fu, S. Rapid assembly of diverse and potent allosteric Akt inhibitors. Bioorg. Med. Chem. Lett. 2008, 18, 2211-2214. [CrossRef] [PubMed]

11. Lindsley, C.W.; Zhao, Z.; Leister, W.H. Allosteric Akt (PKB) inhibitors: Discovery and SAR of isozyme selective inhibitors. Bioorg. Med. Chem. Lett. 2005, 15, 761-764. [CrossRef] [PubMed]

12. Khodarahmi, G.A.; Chen, C.S.; Hakimelahi, G.H. Design, synthesis, and cytotoxicity of 4-sulfonamide substituted benzamidobenzimidazolones and an acyl benzimidazolone. J. Iran. Chem. Soc. 2005, 2, 124-134. [CrossRef]

13. Ballante, F.; Caroli, A.; Wickersham, R.B. Hsp90 inhibitors, part 1: Definition of 3-D QSAutogrid/R models as a tool for virtual screening. J. Chem. Inf. Model. 2014, 54, 956-969. [CrossRef] [PubMed]

14. Rzasa, R.M.; Kaller, M.R.; Liu, G. Structure-activity relationships of 3,4-dihydro-1H-quinazolin-2-one derivatives as potential CDK5 inhibitors. Bioorg. Med. Chem. 2007, 15, 6574-6595. [CrossRef] [PubMed]

15. Monovich, L.; Mugrage, B.; Quadros, E. Optimization of halopemide for phospholipase D2 inhibition. Bioorg. Med. Chem. Lett. 2007, 17, 2310-2311. [CrossRef] [PubMed]

16. Scott, S.A.; Selvy, P.E.; Buck, J.R. Design of isoform-selective phospholipase D inhibitors that modulate cancer cell invasiveness. Nat. Chem. Biol. 2009, 5, 108-117.

17. Lewis, J.A.; Scott, S.A.; Lavieri, R. Design and synthesis of isoform-selective phospholipase D (PLD) inhibitors. Part I: Impact of alternative halogenated privilegedstructures for PLD1 specificity. Bioorg. Med. Chem. Lett. 2009, 19, 1916-1920. [PubMed]

18. Dwyer, M.; Keertikar, P.K.; Paruch, K. Discovery of pyrazolo[1,5-a]pyrimidine-based Piminhibitors: A template-based approach. Bioorg. Med. Chem. Lett. 2013, 23, 6178-6182. [CrossRef] [PubMed]

19. Collins, J.C.; Armstrong, A.; Chapman, K.L. Prospective use of molecular field points in ligand-based virtual screening: Efficient identification of new reversible Cdc25 inhibitors. Med. Chem. Commun. 2013, 4, 1148-1155. [CrossRef]

20. Furber, M.; Alcaraz, L.; Luckhurst, C. Discovery and evolution of phenoxypiperidinehydroxyamide dual CCR3/H1 antagonists. Part I. Bioorg. Med. Chem. Lett. 2012, 22, 7702-7706. [CrossRef] [PubMed] 
21. Li, Q.; Li, T.; Woods, K.W. Benzimidazolones and indoles as non-thiol farnesyltransferase inhibitors based on tipifarnib scaffold: Synthesis and activity. Bioorg. Med. Chem. Lett. 2005, 15, 2918-2922. [CrossRef] [PubMed]

22. Steve, Y.; Cho, E.; Cynthia, M. Plasma and cerebrospinal fluid pharmacokinetics of intravenously administered ABT-751 in non-human primates. Cancer Chemother. Pharmacol. 2007, 4, 563-567.

Sample Availability: Samples of all the compounds are available from the authors.

(C) 2016 by the authors; licensee MDPI, Basel, Switzerland. This article is an open access article distributed under the terms and conditions of the Creative Commons Attribution (CC-BY) license (http://creativecommons.org/licenses/by/4.0/). 